Draft Version July 10, 2018

Preprint typeset using $\mathrm{LAT}_{\mathrm{E}} \mathrm{X}$ style emulateapj v. 04/17/13

\title{
FOMALHAUT B AS A DUST CLOUD: FREQUENT COLLISIONS WITHIN THE FOMALHAUT DISK
}

\author{
S. M. Lawler ${ }^{12}$, S. Greenstreet ${ }^{3}$, And B. Gladman ${ }^{3}$ \\ Draft version July 10, 2018
}

\begin{abstract}
The planet candidate Fomalhaut b is bright in optical light but undetected in longer wavelengths, requiring a large, reflective dust cloud. The most recent observations find an extremely eccentric orbit $(e \sim 0.8)$, indicating that Fomalhaut b cannot be the planet that is constraining the system's eccentric debris ring. An irregular satellite swarm around a super-Earth has been proposed, however, explaining the well-constrained debris ring requires an additional planet on an orbit that crosses that of the putative super-Earth. This paper expands upon a second theory: Fomalhaut b is a transient dust cloud produced by a catastrophic collision between planetesimals in the disk. We perform collisional probability simulations of the Fomalhaut debris disk based on the structure of our Kuiper belt, finding that the catastrophic disruption rate of $d \simeq 100 \mathrm{~km}$ bodies in the high-eccentricity scattering component is several per decade. This model paints a picture of the Fomalhaut system as having recently (within $\sim 10-100 \mathrm{Myr}$ ) experienced a dynamical instability within its planetary system, which scattered a massive number of planetesimals onto large, high eccentricity orbits similar to that of Fom b. If Fomalhaut b is indeed a dust cloud produced by such a collision, we should soon see another appear, while Fomalhaut b will expand until it is either resolved or becomes too faint to be seen.
\end{abstract}

\section{INTRODUCTION}

Fomalhaut is a nearby $(7.70 \mathrm{pc})$, widely-spaced triple star system (Mamajek et al. 2013) with an age of 440 Myr (Mamajek 2012). Fomalhaut A (Fomalhaut hereafter), is an A4 star that dominates the system and possesses a beautiful, eccentric debris ring that is resolved at optical (Kalas et al. 2005), infrared (Faiardo-Acosta et al. 1998; Stapelfeldt et al. 2004; Su et al. 2013; Acke et al. 2012), submillimeter (Holland et al. 1998, 2003; Marsh et al. 2005), and millimeter wavelengths (Boley et al. 2012).

Fomalhaut b was directly imaged using the Hubble Space Telescope (Kalas et al. 2008) and, based on two observation epochs, plausibly consistent with orbital predictions for a massive planet dynamically constraining the eccentric dust ring (Quillen 2006; Chiang et al. 2009). However, follow-up observations in infrared wavelengths failed to detect Fom b (Marengo et al. 2009; Janson et al. 2012), indicating a significantly lower mass than Jupiter.

The nature of Fom b is uncertain. The most recent observations of Fom b suggest that it is on a highly eccentric, possibly ring-crossing orbit Kalas et al. 2013; Beust et al. 2014). Fom b is also brighter at optical wavelengths than predicted by planetary atmosphere models, requiring a cloud of optically reflective small dust grains. Kennedy and Wyatt (2011) suggest a swarm of collisionally grinding irregular satellites, gravitationally bound to a super-Earth planet. However, dynamical analyses suggest that a planet of this mass on such an eccentric orbit will destroy the apsidally aligned debris ring within a

\footnotetext{
${ }^{1}$ Department of Physics and Astronomy, University of Victoria, PO Box 1700, STN CSC Victoria, BC V8W 2Y2, Canada

${ }^{2}$ NRC-Herzberg, 5071 West Saanich Road., Victoria, BC V9E 2E7, Canada

3 Department of Physics and Astronomy, University of British Columbia, 6224 Agricultural Road Vancouver, BC V6T 1Z1, Canada
}

very small fraction $(<1 \%)$ of the stellar age (Kalas et al. 2013; Tamayo 2014; Beust et al. 2014).

Several papers have discussed an alternate option, that Fom b is a post-collision dust cloud (Currie et al. 2012; Galicher et al. 2013; Kalas et al. 2013; Tamavo 2014; Kenvon et al. 2014), and each dismissed this theory because the collisional timescales between planetesimals appears to be uncomfortably long. However, examination of our own Kuiper Belt's structure and an understanding of the clearing of our own Solar System suggests that a significant, unaccounted for, high-eccentricity component may be present.

In this paper, we show that Fom $b$ is plausibly a collision-generated dust cloud, finding a catastrophic disruption rate for objects on Fom b-like orbits high enough that observing a post-collision dust cloud is tenable.

\section{COMPARISON WITH THE KUIPER BELT}

As Kalas et al. (2013) point out, Fomalhaut's debris ring and our Solar System's main Kuiper belt are similar dynamically, despite their different ages. A perhaps important difference is that while the classical Kuiper belt has very low eccentricity, the Fomalhaut debris ring has a significant forced eccentricity of $e \simeq 0.1$. Looking at the large (diameter $d \gtrsim 100 \mathrm{~km}$ ) objects in the Kuiper belt, the only apparent structure is the dense, relatively narrow ring of the main classical belt (Lawler 2014). Due to the high optical depth of the dust grains in the Fomalhaut ring ( $\sim 0^{-3}$; Marsh et al. 2005) compared to the Kuiper Belt $\left(\sim 10^{-7}\right)$, the maximal dust distribution should co-located with the ring (see simulations by Kuchner and Stark 2010).

Fom b's orbit is placed in context in Figure1. where the Kuiper belt, as measured by the Canada-France Ecliptic Plane Survey (CFEPS; Petit et al. 2011; Gladman et al. 2012) had its semimajor axis multiplied by three to match the Fomalhaut system. Though many KBO dynamical sub-classes exist, here (Figure 1) we only dis- 


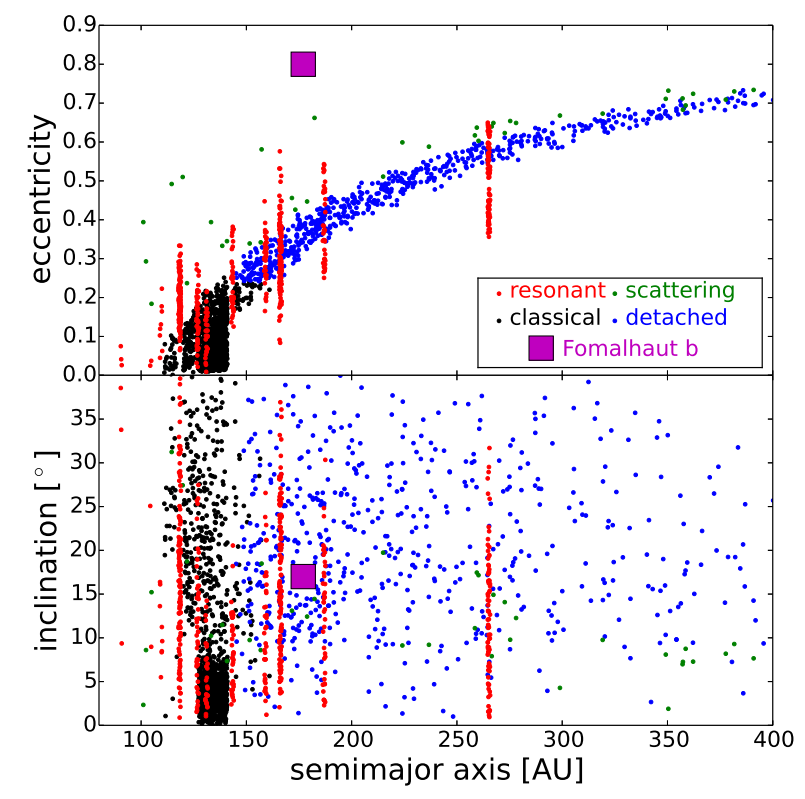

FIG. 1.- Orbital element distributions for the debiased Kuiper Belt as measured by CFEPS, where the semimajor axis has been scaled so that the classical KBOs match Fomalhaut's main debris ring. The different populations are shown by different colors, labelled in the legend. Also shown on the plot is the best-fit orbital elements for Fom b (Kalas et al. 2013, large purple square). Fomalhaut $\mathrm{b}$ most clearly belongs in the scattering population.

cuss four broad categories: resonant objects (red) which are in mean-motion resonances with Neptune, scattering objects (green) whose orbits significantly change due to close encounters with the giant planets within a few Myr, detached objects (blue) which have eccentricities correlated with their semimajor axes, implying past planetary interactions, and lastly classical objects (black) which include the rest of the KBOs (see Gladman et al. 2008). The resonant semimajor axes in the Kuiper Belt depend on the location of Neptune, thus we do not expect to find objects in these exact locations in the Fomalhaut disk.

With a pericenter well inside the ring, Fom b is clearly a member of the scattering population. Our hypothesis is that Fom b is a newly disrupted member of Fomalhaut's scattering planetesimal population, in a recently perturbed Fomalhaut system, similar to the Nice Model instability that occurred early in our Solar System's history.

\section{MODELLING THE FOMALHAUT DISK}

Our model of the Fomalhaut disk is based on the known structure and populations of the Kuiper Belt. The Kuiper Belt's scattering disk in particular is heavily eroded from its state just after the migration of the giant planets. Simulations show that today's scattering population is $\sim 1 \%$ of the original (Duncan and Levison 1997), which has been eroded away mainly by planetary close encounters causing ejection from the Solar System. Because Fomalhaut is significantly younger than the Sun, in order to transform our Kuiper Belt into an approximation of the Fomalhaut disk, we first must correct for this erosion. This brings the number of scattering objects to about the same as the classical population, though in a much more widely dispersed orbital distribution. Addi- tionally, very massive planets $\left(\gtrsim 5 M_{\text {Jup }}\right)$ with projected separations $>15$ AU have been ruled out (Currie et al. 2013), so the timescale for depletion of the scattering population shouldn't be much faster than in our Solar System. Thus, the number of scattering objects is scaled up by 100 times relative to other Kuiper Belt populations.

The orbital distribution of objects in the young scattering disk was different from today's distribution, as some orbits are more likely to be ejected than others. We produced a simple "young scattering" distribution for our Solar System by starting with a flat distribution of objects with $a=4-35 \mathrm{AU}, e=0.1$, and $i=15^{\circ}$, and running a numerical integration with the four giant planets for $100 \mathrm{Myr}$. The surviving objects, many of which show more deeply crossing perihelia than for the scattering population shown in Figure 1, had their semimajor axes tripled to match the Fomalhaut system.

Next, one must also scale up the system mass until the main classical belt matches the Fomalhaut ring's much larger observed mass compared to our Kuiper Belt. CFEPS measured the absolute number of $d \gtrsim 100 \mathrm{~km}$ Kuiper Belt objects, so matching the mass of another disk is accomplished by scaling the number of $d>100 \mathrm{~km}$ objects in the model. Coincidentally, $d \simeq 100 \mathrm{~km}$ is the minimum size required to produce the observed Fom b dust cloud (Galicher et al. 2013; Kenvon et al. 2014). We settled on a scaling factor of 1000 . This is very approximate, as mass estimates for the Kuiper Belt vary by an order of magnitude $\left(\sim 0.01-0.1 M_{\oplus}\right.$; e.g., Fraser et al. 2014; Vitense et al. 2010), and mass estimates for the Fomalhaut ring vary by two orders of magnitude $(\sim 2$ $110 M_{\oplus}$; e.g., Boley et al. 2012; Acke et al. 2012).

Orbital elements for simulated planetesimals in the Fomalhaut model are based on the CFEPS L7 synthetic model of the Kuiper Belt (available at www.cfeps.net). The heart of the classical Kuiper Belt sits at $\sim 45 \mathrm{AU}$, while the peak of the Fomalhaut disk as measured by ALMA (Bolev et al. 2012) is at 135 AU, so we multiplied the semimajor axes of each synthetic object by three. To match the main ring shape and higher eccentricity, the eccentricities of the main classical belt were increased by 0.05 , so that the median eccentricity became $e \simeq 0.1$. To match the apsidal aligment of the planetesimals relative to each other, the simulated classical objects were set with $\Omega=-\omega \pm 10^{\circ}$.

This orbital distribution of synthetic objects is the starting point for our collisional probability simulations.

\subsection{Collisional Probability Simulations of the Fomalhaut System}

We use an Opik collision probability code based on Dones et al. (1999) to compute the collision probability for a Fom b progenitor (radius $r=50 \mathrm{~km}, a=177 \mathrm{AU}$, $e=0.8, i=17^{\circ}$ ) with various small body populations, ignoring any other planets in the system. The code calculates the collision probability for a population of projectiles by numerically integrating over the precession cycle of the nodal longitude $\Omega$ and argument of pericenter $\omega$ for both the impactor and target bodies.

The code was modified to bin the collision probability into individual impact velocity bins (as opposed to binning the total collision probability into an average impact velocity bin computed from all possible impact orienta- 
tions over a full precession cycle of the orbital nodes), as well as individual impact distance bins. This produces detailed impact velocity distributions for each projectile population.

\subsection{Catastrophic Collisional Probability}

Using the probabilities for a range of impact velocities in each distance bin, we calculate the total catastrophic disruption probability. Closer to the star, relative speeds are generally higher allowing smaller objects to produce the same collision energy as a slower, larger object farther from the star.

Equation 2 from Leinhardt and Stewart (2012) relates target mass, projectile mass, collisional velocity, and the catastrophic disruption energy for an icy body (which we take from Figure 11 in Leinhardt and Stewart 2009, using $r=50 \mathrm{~km}$ ). This gives the minimum projectile size required to catastrophically disrupt a $d=100 \mathrm{~km}$ target body at a given velocity. To properly weight the collisional probabilities in each velocity bin, we multiply by the number of bodies of this minimum size, assuming a projectile size distribution of $d n / d r \propto r^{-q}$, with $q=3.5$, as for a collisional cascade (Dohnanvi 1969), consistent with other estimates of the disk mass (e.g., Boley et al. 2012; Acke et al. 2012) from the literature.

Figure 2 shows the catastrophic collision probability distribution per year per $2 \mathrm{AU}$-wide bin, for three different projectile populations. Integrating over each curve gives the total probability per year of a given population catastrophically disrupting a $d=100 \mathrm{~km}$ progenitor on a Fom b-like orbit.

The full disk collisional model (black line in Figure 2) shows the likelihood of collisions between an object on a Fom b-like orbit and anything in the Fomalhaut system model. The total integrated catastrophic collisional probability is 14 /decade, and the most likely place for collisions is just sunward of the main ring. The portion of the catastrophic probability for just the non-scattering objects is also shown separately (red line); as expected the main ring dominates at its distance, but most scattering object disruptions are mutual and occur between 50-110 AU. We also confirmed that our model produces a catastrophic disruption rate for collisions between objects in the main ring that approximately reproduces the observed dust production rate (Acke et al. 2012).

Though the probabilities for disruption of a Fom b-like orbit are high within the main belt, collisions here would not generate a dust cloud because the high dust density within the ring would effectively absorb and/or collisionally destroy the dust cloud almost instantly. Also, due to Fom b's orbital direction, we know Fom b has not been inside the main belt for at least $\sim 250$ years. Therefore, this is not our favoured projectile population.

The most likely population to cause a catastrophic collision is the scattering population (blue line in Figure 2). This curve peaks at about 95 AU from Fomalhaut, similar to Fom b's distance $~ 10-15$ years ago, with a total integrated catastrophic collision rate for $d=100 \mathrm{~km}$ objects of $11 /$ decade. The most likely collision is a $\sim 10 \mathrm{~km}$ projectile destroying a $100 \mathrm{~km}$ target.

\section{PLAUSIBILITY OF FOM B AS A DUST CLOUD}

Galicher et al. (2013) estimate that within the main belt, mutual collisions between $d=100 \mathrm{~km}$ bodies

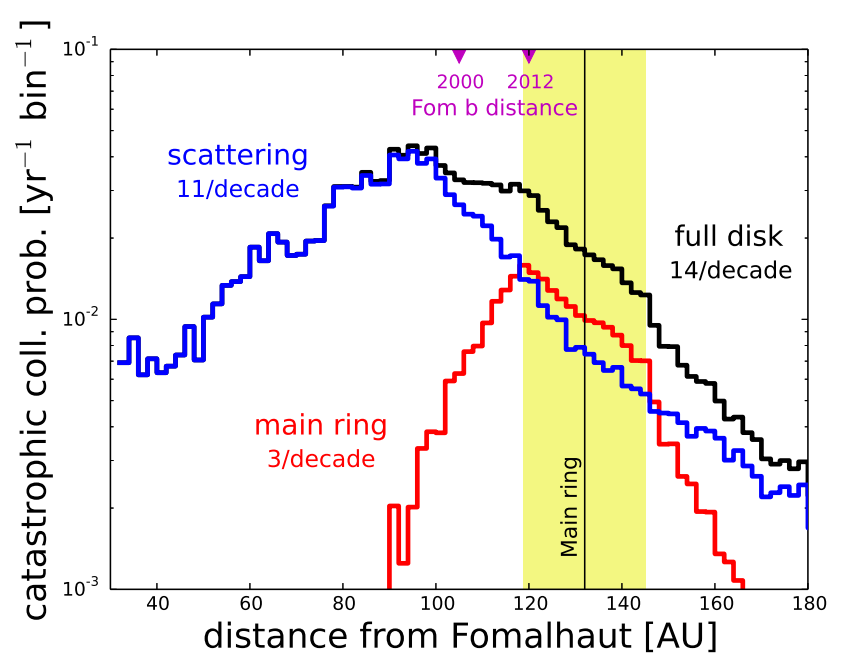

FIG. 2.- The catastrophic collision probability per year per 2 AU-wide bin for different projectile populations onto a target with Fom b's current orbit. Black shows the full disk model, red shows just the non-scattering component (the main ring), and blue shows just the scattering component, with total integrated catastrophic disruption probabilities stated for each population. Yellow band shows the pericenter-apocenter range of main ring particles, the mutual main ring collision rate (not shown) is a factor of several larger. Measured stellocentric distances of Fom b in 2000 and 2012 are shown.

happen once per century (they do not calculate catastrophic disruption rate), while Currie et al. (2012) and Kalas et al. (2013) argue that the dust cloud scenario is unlikely because of the long collisional timescale and relatively quick orbital shearing. Our analysis shows that closer to the star than the main ring, higher mutual collision speeds result in smaller objects being able to catastrophically disrupt the same size target. Because of the size distribution, there are abundantly more smaller objects, which shifts the peak destruction probability sunward of the main ring.

Kenvon et al. (2014) use size distribution models to argue that a dust cloud resulting from a collision is only possible for a limited range of parameters. A $50 \mathrm{~km}$ radius progenitor can reproduce the observed properties for a dust power-law size index $(q)$ of 4.7 or less, with the largest remaining fragment less than $1 \%$ the size of the initial body (otherwise there is not enough mass in dust to reproduce observations). While similar $q$ values are often reproduced in simulations of catastrophic disruptions, near-complete destruction of the target is only rarely produced in simulations (Leinhardt and Stewart 2012). Additionally, Kenyon et al. (2014) point out that the expansion of the dust cloud is constrained by the eight year observational baseline. If the dust cloud is unresolved (Currie et al. 2012; Kalas et al. 2013; Kenyon et al. 2014), this limits the expansion to $\delta 300 \mathrm{~m} / \mathrm{s}$, while if it is marginally resolved (Galicher et al. 2013), the expansion is limited to $\lesssim 1 \mathrm{~km} / \mathrm{s}$. Both of these values are significantly less than the relative velocities for the bulk of simulated collisions.

However, as discussed in Kenvon et al. (2014), the expansion rate of a dust cloud resulting from a catastrophic collision onto a fairly large body $(\sim 100 \mathrm{~km})$ depends not on the collision velocity, but on the escape velocity of the total mass (projectile+target; Benz and Asphaug 1999). 
For a $d=100 \mathrm{~km}$ icy body, the escape velocity is approximately $30 \mathrm{~m} / \mathrm{s}$, suggesting that there is close to a century before the dust cloud would expand enough to be resolved by Hubble, greatly increasing the window of time the dust cloud remains unresolved.

This theory implies that there are many possibly visible dust clouds in the Fomalhaut system at any given time. However, we note the difficulty of these observations: only a limited range of distances and longitudes have deep enough magnitude limits to detect such a cloud (Kalas et al. 2013; Currie et al. 2012). Also, only a fraction of the catastrophic collisions will result in a visible dust cloud (Kenvon et al. 2014). Lastly, 11/decade is probably an overestimate since our scattering population estimate is appropriate for just after the system's perturbation; if instead the perturbation occurred 30 Myr ago (perhaps a more reasonable $\sim 10 \%$ of the system's age) our integrations show that the catastrophic collision rate for the scattering population would drop to $\sim 1$ /decade as the unseen planets gradually eject planetesimals.

A recent example of an observed expanding cloud involves Comet 17P/Holmes, which outburst in 2007 resulting in an optically bright, nearly spherical cloud of dust. While this outburst was most likely due to a gas-pressure driven explosion and not a collision (e.g., Stevenson et al. 2010), it provides a well-studied analogue to the sort of expanding dust cloud we propose to explain Fom b. The cloud of Comet Holmes was roughly divided into two parts with similar masses that expanded at different rates (Reach et al. 2010): the smallest $(\sim 2 \mu \mathrm{m})$ grains expanded the fastest $(\sim 250 \mathrm{~m} / \mathrm{s})$, creating a large shell that was the most easily visible, and a second cloud composed of larger $(\sim 200 \mu \mathrm{m})$ grains expanding more slowly $(\sim 9 \mathrm{~m} / \mathrm{s})$. Because of Fomalhaut's higher luminosity, the blowout limit $(\sim 10 \mu \mathrm{m}$ for icy grains) is much larger than in our Solar System. Any grains smaller than this would immediately be placed on hyperbolic orbits. The relative fraction of large versus small dust grains produced by the collision would dictate which portion would be more easily visible: either the larger grains that continue on roughly the same orbit as the parent body, or the small grains which find themselves on either an extremely elliptical or even hyperbolic orbit.

The latter offers another possible explanation for the extreme orbit of Fom b, as small grains with high $\beta$ values will instantaneously have their orbits shift to high $e$. While Beust et al. (2014) rule out $e>0.98$ in their orbital fits for Fom b, Kalas et al. (2013) do not specifically rule out unbound orbits. If Fom b's orbit is found to be unbound in future observations, a dust cloud made of small grains is a more plausible explanation than an extremely fortuitous observation of a planet being ejected from the system.

\section{DISCUSSION: EVOLUTION OF THE FOMALHAUT SYSTEM}

The theory that Fom b is a collisional dust cloud fits into the following possible narrative of the Fomalhaut system. Fomalhaut hosts a young planetary system. As has been proposed for the early few hundred Myr of our own Solar System (Gomes et al. 2005), the Fomalhaut system experienced a relatively recent $(\sim 100 \mathrm{Myr})$ dynamical instability that resulted in a dramatic reshuffling of the orbits of its planets and smaller bodies. Simulations of our own Solar System show that a dynamically cold population (like the debris ring) can survive such a violent event (Batygin et al. 2011). Fomalhaut c (as yet unobserved) was scattered or migrated onto its current moderately eccentric orbit $(e \simeq 0.1$ ) and is currently secularly driving the eccentricity of the main debris ring, as discussed in previous works. Any planetesimals that were on orbits interior to the main ring $(a \simeq 135)$ were scattered, and this scattering population is currently being eroded by close encounters with Fom c and any other planets interior to Fom c. The erosion rate of this population depends on the masses and orbital configurations of the planetary system: larger planets will erode the scattering disk faster, as will more closely spaced planets, since they are more easily able to "hand off" scattered planetesimals to each other.

Due to the low surface density of this scattering population relative to the main ring, a belt of dust resulting from mutual collisions within this population would not be as easily visible, and indeed much of it could be ejected from the system immediately after formation due to high $\beta$ values (e.g., Wyatt 2008). Thus, Fomalhaut could be hiding a significant population of KBO-analogues on highly eccentric orbits similar to that of Fomalhaut b. More detailed modelling is required to determine the orbital population and spectral energy distribution of dust resulting from these collisions within the scattering population. A likely outcome would be a population of warm dust grains, as found by recent IR observations ( $\mathrm{Su}$ et al. 2013; Acke et al. 2012).

There are two predictions of our Fom b-as-a-dust-cloud hypothesis. The first is that the $\sim 30-100 \mathrm{~m} / \mathrm{s}$ Fom $\mathrm{b}$ expansion over the coming decades will eventually permit the object to be resolved. The second prediction is that more Fom b-like dust clouds should be created in the system in the near future.

\section{SUMMARY AND CONCLUSION}

We have shown that the catastrophic disruption rates within the Fomalhaut debris disk can be much higher than previously assumed. This is primarily due to inclusion of a high eccentricity scattering component, equivalent to the scattering population in our Solar System's Kuiper Belt. Fomalhaut's young age is also an important factor permitting a more massive scattering component. This calculation shows that the possibility that Fom b is just a cloud of dust should not be dismissed.

The relatively high collision rate that we calculate here would mean that another Fom b-like object should appear within the next decade, and Fom b itself will fade over the coming years, possibly becoming resolved. In order to test these two predictions, continued follow-up observations capable of detecting objects as faint as Fom b are vital. For now, the only telescope capable of detecting Fom b is Hubble, but the upcoming James Webb Space Telescope will be able to resolve the dust cloud, and provide some additional constraints on the dust composition with near-IR measurements.

While perhaps disappointing to think of Fom b as merely a cloud of dust and not an actual planet, this scenario tells us about the structure of the Fomalhaut debris disk, and strongly implies the presence of multiple planets, just as the scattering component in our Solar 
System is constrained and limited by the presence of giant planets interior to the Kuiper Belt.

S.M.L. wishes to thank Wes Fraser, Kat Volk, Aaron Boley, Yanqin Wu, Dan Tamayo, Christa Van Laerhoven, and Henry Ngo for enlightening discussions and advice that made this project possible. S.M.L. acknowledges an NSERC Discovery Accelerator Supplement which funded this work.

\section{REFERENCES}

Acke, B., Min, M., Dominik, C., Vandenbussche, B., Sibthorpe, B., Waelkens, C., Olofsson, G., Degroote, P., Smolders, K., Pantin, E., Barlow, M. J., Blommaert, J. A. D. L., Brandeker, A., De Meester, W., Dent, W. R. F., Exter, K., Di Francesco, J., Fridlund, M., Gear, W. K., Glauser, A. M., Greaves, J. S., Harvey, P. M., Henning, T., Hogerheijde, M. R., Holland, W. S., Huygen, R., Ivison, R. J., Jean, C., Liseau, R., Naylor, D. A., Pilbratt, G. L., Polehampton, E. T., Regibo, S., Royer, P., Sicilia-Aguilar, A., and Swinyard, B. M.: 2012, A\&\&A 540, A125

Batygin, K., Brown, M. E., and Fraser, W. C.: 2011, ApJ 738, 13

Benz, W. and Asphaug, E.: 1999, Icarus 142, 5

Beust, H., Augereau, J.-C., Bonsor, A., Graham, J. R., Kalas, P., Lebreton, J., Lagrange, A.-M., Ertel, S., Faramaz, V., and Thébault, P.: 2014, A $6 A$ 561, A43

Boley, A. C., Payne, M. J., Corder, S., Dent, W. R. F., Ford, E. B., and Shabram, M.: 2012, ApJ 750, L21

Chiang, E., Kite, E., Kalas, P., Graham, J. R., and Clampin, M.: 2009, ApJ 693, 734

Currie, T., Cloutier, R., Debes, J. H., Kenyon, S. J., and Kaisler, D.: 2013, ApJ 777, L6

Currie, T., Debes, J., Rodigas, T. J., Burrows, A., Itoh, Y., Fukagawa, M., Kenyon, S. J., Kuchner, M., and Matsumura, S.: 2012, ApJ 760, L32

Dohnanyi, J. S.: 1969, J. Geophys. Res. 74, 2531

Dones, L., Gladman, B., Melosh, H. J., Tonks, W. B., Levison, H. F., and Duncan, M.: 1999, Icarus 142, 509

Duncan, M. J. and Levison, H. F.: 1997, Science 276, 1670

Fajardo-Acosta, S. B., Telesco, C. M., and Knacke, R. F.: 1998, AJ 115, 2101

Fraser, W. C., Brown, M. E., Morbidelli, A., Parker, A., and Batygin, K.: 2014, ApJ 782, 100

Galicher, R., Marois, C., Zuckerman, B., and Macintosh, B.: 2013, ApJ 769, 42

Gladman, B., Lawler, S. M., Petit, J.-M., Kavelaars, J., Jones, R. L., Parker, J. W., Van Laerhoven, C., Nicholson, P., Rousselot, P., Bieryla, A., and Ashby, M. L. N.: 2012, AJ 144, 23

Gladman, B., Marsden, B. G., and Vanlaerhoven, C.: 2008, Nomenclature in the Outer Solar System, pp 43-57

Gomes, R., Levison, H. F., Tsiganis, K., and Morbidelli, A.: 2005 , Nature 435, 466

Holland, W. S., Greaves, J. S., Dent, W. R. F., Wyatt, M. C., Zuckerman, B., Webb, R. A., McCarthy, C., Coulson, I. M., Robson, E. I., and Gear, W. K.: 2003, ApJ 582, 1141

Holland, W. S., Greaves, J. S., Zuckerman, B., Webb, R. A., McCarthy, C., Coulson, I. M., Walther, D. M., Dent, W. R. F., Gear, W. K., and Robson, I.: 1998, Nature 392, 788
Janson, M., Carson, J. C., Lafrenière, D., Spiegel, D. S., Bent, J. R., and Wong, P.: 2012, ApJ 747, 116

Kalas, P., Graham, J. R., Chiang, E., Fitzgerald, M. P., Clampin, M., Kite, E. S., Stapelfeldt, K., Marois, C., and Krist, J.: 2008, Science 322, 1345

Kalas, P., Graham, J. R., and Clampin, M.: 2005, Nature 435, 1067

Kalas, P., Graham, J. R., Fitzgerald, M. P., and Clampin, M.: 2013, ApJ 775, 56

Kennedy, G. M. and Wyatt, M. C.: 2011, MNRAS 412, 2137

Kenyon, S. J., Currie, T., and Bromley, B. C.: 2014, ApJ 786, 70

Kuchner, M. J. and Stark, C. C.: 2010, AJ 140, 1007

Lawler, S. M.: 2014, in M. Booth, B. C. Matthews, and J. R. Graham (eds.), IAU Symposium, Vol. 299 of IAU Symposium, pp 232-236

Leinhardt, Z. M. and Stewart, S. T.: 2009, Icarus 199, 542

Leinhardt, Z. M. and Stewart, S. T.: 2012, ApJ 745, 79

Mamajek, E. E.: 2012, ApJ 754, L20

Mamajek, E. E., Bartlett, J. L., Seifahrt, A., Henry, T. J., Dieterich, S. B., Lurie, J. C., Kenworthy, M. A., Jao, W.-C., Riedel, A. R., Subasavage, J. P., Winters, J. G., Finch, C. T., Ianna, P. A., and Bean, J.: 2013, ArXiv e-prints

Marengo, M., Stapelfeldt, K., Werner, M. W., Hora, J. L., Fazio, G. G., Schuster, M. T., Carson, J. C., and Megeath, S. T.: 2009, ApJ 700, 1647

Marsh, K. A., Velusamy, T., Dowell, C. D., Grogan, K., and Beichman, C. A.: 2005, ApJ 620, L47

Petit, J., Kavelaars, J. J., Gladman, B., Jones, R. L., Parker, J. W., Van Laerhoven, C., Nicholson, P., Mars, G., Rousselot, P., Mousis, O., Marsden, B., Bieryla, A., Murray, I., Ashby, M. L. N., Benavidez, P., Campo Bagatin, A., and Veillet, C.: 2011, AJ

Quillen, A. C.: 2006, MNRAS 372, L14

Reach, W. T., Vaubaillon, J., Lisse, C. M., Holloway, M., and Rho, J.: 2010, Icarus 208, 276

Stapelfeldt, K. R., Holmes, E. K., Chen, C., Rieke, G. H., Su, K. Y. L., Hines, D. C., Werner, M. W., Beichman, C. A., Jura, M., Padgett, D. L., Stansberry, J. A., Bendo, G., Cadien, J., Marengo, M., Thompson, T., Velusamy, T., Backus, C., Blaylock, M., Egami, E., Engelbracht, C. W., Frayer, D. T., Gordon, K. D., Keene, J., Latter, W. B., Megeath, T., Misselt, K., Morrison, J. E., Muzerolle, J., Noriega-Crespo, A., Van Cleve, J., and Young, E. T.: 2004, ApJS 154, 458

Stevenson, R., Kleyna, J., and Jewitt, D.: 2010, $A J$ 139, 2230

Su, K. Y. L., Rieke, G. H., Malhotra, R., Stapelfeldt, K. R., Hughes, A. M., Bonsor, A., Wilner, D. J., Balog, Z., Watson, D. M., Werner, M. W., and Misselt, K. A.: 2013, ApJ 763, 118 Tamayo, D.: 2014, MNRAS 438, 3577

Vitense, C., Krivov, A. V., and Löhne, T.: 2010, A $\dot{\xi} A$ 520, A32+ Wyatt, M. C.: 2008, ARAE A 46, 339 